\title{
Defeat Depression Campaign
}

\section{Attitudes towards depression}

\author{
Christine M. Vize, Department of Psychiatry, St Mary's Hospital Medical School; and \\ R. G. PRIEST, Professor and Head of Department of Psychiatry, St Mary's Hospital \\ Medical School, Praed Street, London W2 1NY
}

The Royal College of Psychiatrists in association with the Royal College of General Practitioners and other relevant organisations launched the Defeat Depression campaign in January 1992. The objectives of the campaign are to reduce the stigma associated with depression, to assist general practitioners and other health care professionals in the recognition and treatment of depressive illness, and to increase public awareness of the extent and treatability of depression.

The Royal Colleges commissioned surveys on attitudes towards depression, to provide a baseline from which changes in attitudes following the five year campaign could later be measured. The pilot surveys were qualitative, with researchers interviewing small samples of the general public, health care professionals (practice nurses, health visitors, pharmacists and social workers), and clinicians (general practitioners, psychiatrists, physicians and paediatricians). These were followed, in December 1991, by a quantitative survey conducted by MORI, in which 2,009 people aged over 15 were interviewed in their homes at 143 sampling points across Great Britain.

\section{Qualitative surveys}

Some interesting attitudes emerged from the initial qualitative studies. Perceived definitions of depression were based on personal experience and articles, particularly in women's magazines. The majority felt that depression could run in families, but that this was an environmental and not a genetic effect, and some 'causes' of depression, such as premenstrual tension or bereavement, rendered the illness more 'legitimate'. There was no consensus as to what constituted a clinical depression, and there was confusion between depression and schizophrenia.

Members of the public said that they would be disinclined to visit their GP complaining about an emotional problem or depression, but would consult with related physical symptoms such as insomnia. Feelings about treatment were mixed. The most commonly mentioned drug associated with the treatment of depression was Valium or 'tranquillisers', and drugs were considered to 'dull' the problem rather than cure it. Counselling was felt to be the most appropriate and effective treatment for depression, as it could get to the 'root' of the problem.

Even among the health care professionals there was a misunderstanding of what depression is, as it was not seen as serious compared with 'psychosis, neurosis and schizophrenia'. Interviews with clinicians did not generate any surprises with respect to the diagnosis of depression, but it was interesting to note that non-psychiatric clinicians, in common with the general public, tended to be unsure of the distinction between a psychologist and a psychiatrist.

\section{MORI quantitative survey December 1991}

MORI used a two-stage sampling design for the quantitative survey. The random sampling points were selected using a system based on parliamentary constituencies across Great Britain. Within each sampling point, respondents were selected according to a quota system of sex, age, social class and whether they were in full-time employment or not. This was done to ensure that the demographic profile of the sample in each region of the country matched the actual profile. The sample was collected by interviewers knocking on people's doors at a variety of different times of day. The data were then weighted to adjust for any discrepancies in the coverage of individual sampling points.

The weighted sample comprised 2003 people, $52 \%$ of whom were female and $48 \%$ male; $38 \%$ were in the 15-34 age group, $29 \%$ in the 35-54 group and 33\% were over 55. Eighteen per cent were in social class $\mathrm{AB}, 22 \%$ in class $\mathrm{Cl}, 28 \%$ in class $\mathrm{C} 2$ and $31 \%$ in class DE. Of the total sample, $22 \%$ reported having suffered from depression $(27 \%$ of women and $17 \%$ of men), and $55 \%$ had either suffered from depression themselves or been in contact with someone who had. 


\section{Findings}

The results showed that the majority of people interviewed believe that depression is an illness, and that anyone can suffer from it. The most common perceived causes are personal and social, although one third think that depression can be caused by 'biological changes in the brain'. Over $90 \%$ of people interviewed consider that people suffering from depression should be offered counselling, whereas only $16 \%$ feel that they should be offered antidepressants. There is no significant difference in this view across different sexes, ages or social classes, or between those who had suffered from depression and those who had not. Surprisingly, there is a view across all subgroups that antidepressants are addictive, with an overall $78 \%$ subscribing to this view (and $87 \%$ believing that tranquillisers are addictive). There was also considerable unanimity between subgroups regarding the efficacy of treatments for depression, with $85 \%$ of those interviewed believing counselling to be effective, as against $46 \%$ for antidepressants and $40 \%$ for tranquillisers. Sixty-two per cent said spontaneously that they would consult their GP if they became depressed, and a further $17 \%$ agreed with this when prompted. However, a majority still felt they would be embarrassed, and half would worry that the GP would regard them as 'unbalanced' or 'neurotic'; $58 \%$ felt that GPs just tend to give out pills for depression.

\section{Comments}

This work, particularly the quantitative survey, offers some guidelines for the Defeat Depression Campaign and for individual practitioners regarding the attitudes of the general public to depression. It was not intended to be a rigorous epidemiological study, but it was still able to demonstrate aspects of the subject which are particularly prone to misconception and misapprehension. It is salutary to note what a high proportion of the general public regard antidepressants as addictive, and that antidepressants and tranquillisers are not perceived as substantially different in their efficacy in treating depression. The vast majority of the sample who favour counselling as the treatment of choice are voicing a view which is entirely consistent with their opinion of the causes of depression. The idea that an illness with a social causation can be effectively treated by chemical means is obviously not very persuasive, although it would be interesting to compare this result to attitudes regarding the use of drugs for anxiety and insomnia.

The clarity of the information obtained from these surveys may be somewhat dimmed as far as psychiatrists are concerned due to non-medical wording of the questions. These attempted to address the issues using lay terminology, but as a result may only mirror public conceptions rather than modifying them. For example, statements such as "depressed people are often mad or mentally unstable" or "depression is not as serious as neurosis or psychosis" would be tendentious to psychiatrists. They demonstrate the difficulties in trying to get a clear idea of attitudes without constraining them by defining the problems in medical terms.

Having obtained an idea of the attitudes of the general public, it is now time to start clarifying misapprehensions, and the best way to start would be to try and ensure that clinicians and the public are talking about the same thing. Clinicians should not make unwarranted assumptions about the knowledge of their patients or the public, and this means addressing basic issues such as the differences between depression and schizophrenia and the concept of 'madness'. Many clinicians will use lay terminology in trying to explain concepts to their patients, but are we just perpetuating misunderstanding by doing this?

The MORI survey did not address possible reasons for the widespread belief that antidepressants are addictive, but from the answers to related questions and from clinical experience it seems likely that many members of the public do not know the difference between antidepressants and benzodiazepines. In the qualitative survey Valium was the most commonly mentioned drug treatment for depression, and $40 \%$ of those questioned in the large survey think tranquillisers are an effective treatment. Should doctors try to correct these misconceptions by stopping the use of the word 'tranquilliser' altogether and describing drugs as antidepressants, hypnotics and anxiolytics or 'anxiety reducing drugs'?

If the Defeat Depression Campaign, together with medical practitioners, could increase the accuracy of public perceptions of depression and its treatment, it would be a large step towards achieving its aim of reducing the stigma associated with a condition which probably half of the population have witnessed or suffered from themselves. 